\title{
QUASI-3D RESISTIVITY IMAGING - MAPPING OF THREE DIMENSIONAL STRUCTURES USING TWO DIMENSIONAL DC RESISTIVITY TECHNIQUES
}

\author{
T. Dahlin', M.H. Loke ${ }^{2}$ \\ 'Dept. of Geotechnology, Lund University, Box 118, S-221 00 Lund, Sweden \\ 'School of Physics, Universiti Sains Malaysia, 11800 USM, Penang, Malaysia

\section{INTRODUCTION}

So far, one dimensional (ID) and two dimensional (2D) techniques have dominated resistivity surveying, and the latter only in recent years, while three dimensional (3D) techniques are still in their infancy. In order to build images of the $3 \mathrm{D}$ resistivity distribution of the ground, it is today common practice to merge the result from a number of sections acquired and inverted using $2 \mathrm{D}$ resistivity imaging techniques, which may be referred to as a quasi-3D technique.

Although 3D techniques will evolve considerably in the coming years, 2D data acquisition techniques will remain preferable for logistical reasons in many cases. It is often a major advantage to carry out the measurements along linear features such as paths, roads etc., whereas it may be impossible to arrange a 3D electrode layout in the terrain in many cases. Strategies based on a relatively sparse net of parallel or crossing 2D sections, that may be filled in with additional sections depending on the preliminary results, may be most efficient in practical applications.

The objective of this study is to assess the effect on $2 \mathrm{D}$ resistivity surveying of $3 \mathrm{D}$ variation in the resistivity structures, and hence the practical applicability of a quasi-3D technique. The work was carried out by means of numerical modelling.

\section{METHOD}

Forward model responses of 3D structures were calculated using numerical modelling, where a finite difference (FD) code was employed (Dey and Morrison 1979; Loke and Barker 1996b). A grid of $31 \mathrm{x}$ 21 electrodes was used for the examples below, with a division of 2 cells between the electrodes.

Thereafter, 2D profiles (or CVES) were extracted from the 3D model responses, with simulated electrode layout in the $x$-direction. Gauss distributed random noise was added to the calculated responses, with $5 \%$ standard deviation in the examples presented here. Four different arrays were tested, Wenner, pole-pole, pole-dipole and dipole-dipole array.

Each profile was inverted individually using a 2D quasi-Newton inversion technique (Loke and Barker 1996a). In the inversion process a FD model is automatically adjusted to fit the input data through a smoothness constrained iterative process. The layer thicknesses were generated automatically by the inversion routine, and differ between the array types, and the forward 3D model.

Finally, the inverted $2 \mathrm{D}$ models were merged to form a quasi-3D model, which was visualised as depth slices. In order to fit the plots on the page, some depth levels have been left out. Due to limited space, only the Wenner and dipole-dipole results are shown here, and only two different models.

\section{EXAMPLE: HIGH RESISTIVE BANDS AT SURFACE}

The example shown in figure I consists of high resistive $(500 \Omega \mathrm{m})$ bands in an otherwise low resistive surrounding $(10 \Omega \mathrm{m})$. The high resistive feature is present in the two top layers, being 0.7 units deep, and the position is marked in the top slice. Below this level the model is homogeneous with a 
MODEL
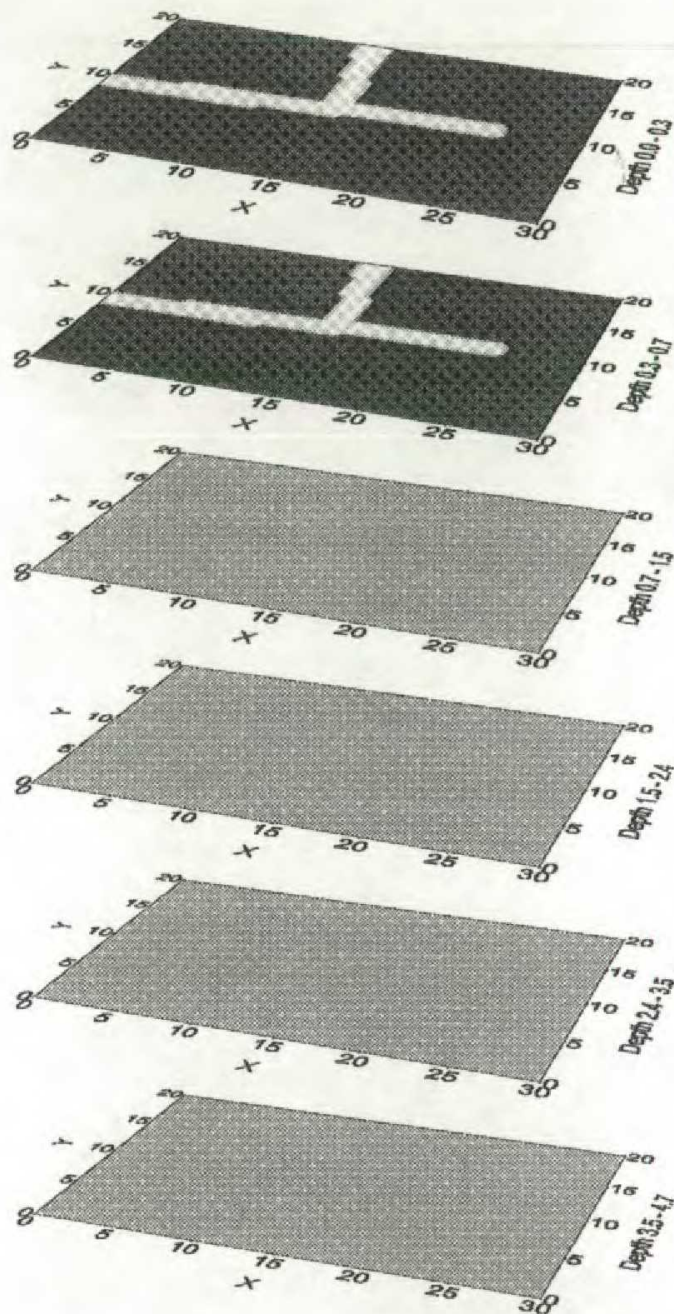

WENNER $3 \mathrm{D} \Rightarrow>2 \mathrm{D} \Rightarrow>3 \mathrm{D}$
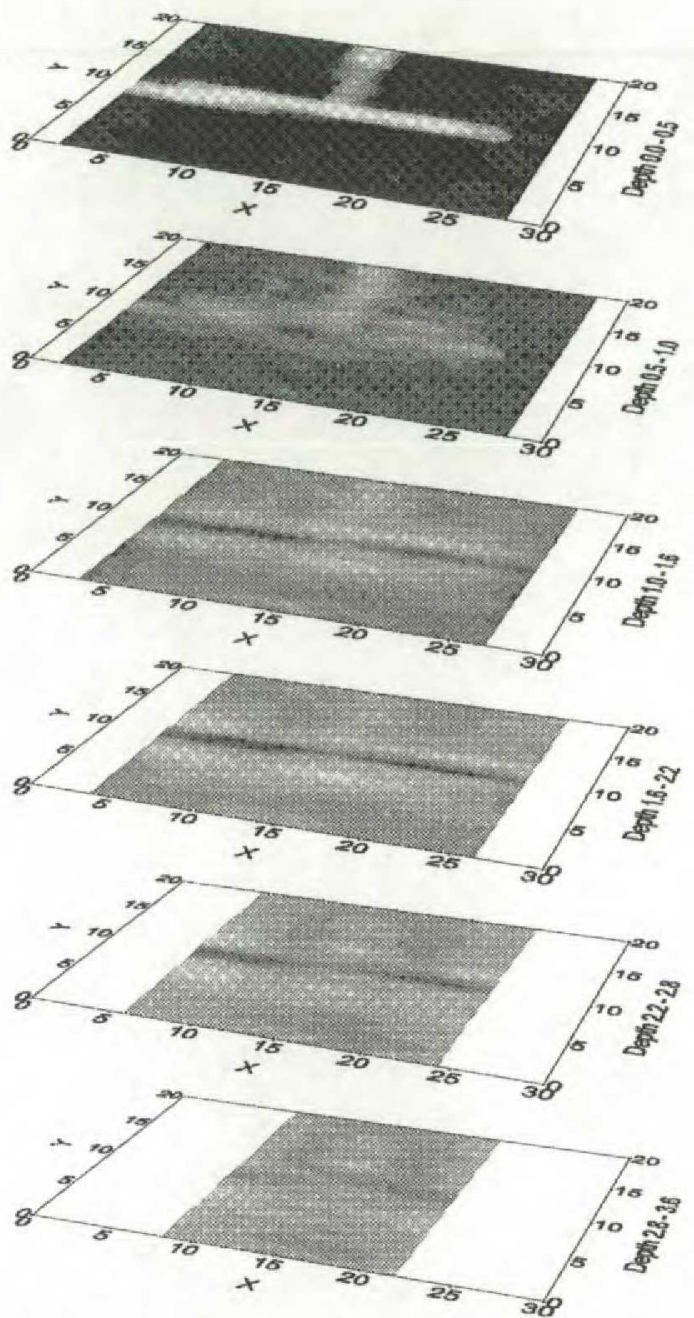

DIPOLE-DIPOLE $3 \mathrm{D}=>2 \mathrm{D}=>3 \mathrm{D}$

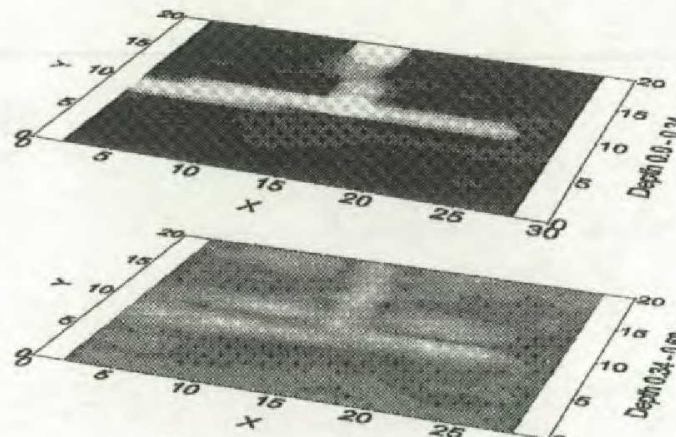

Resistivity

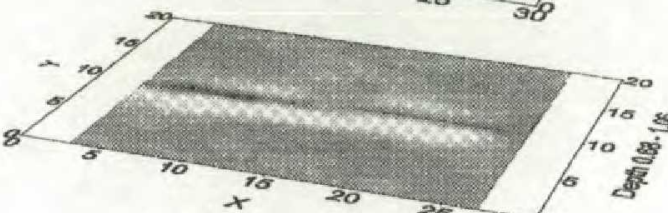

$+300$
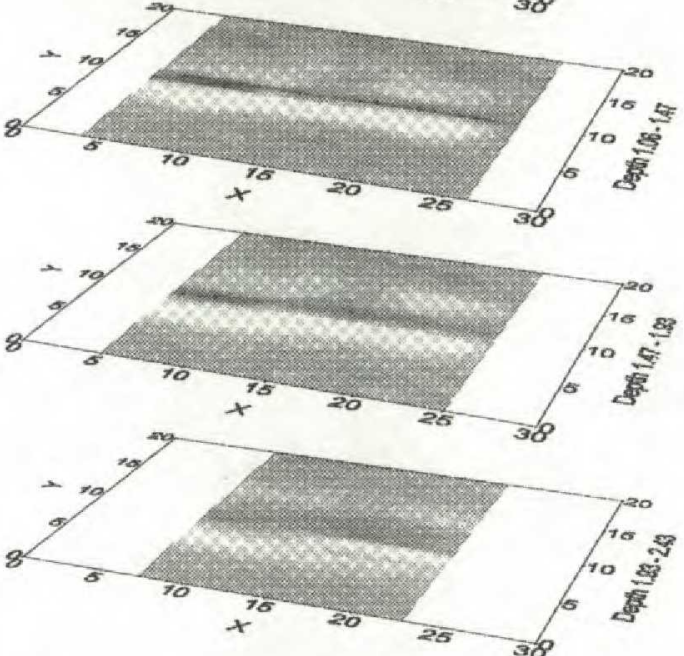

Figure 1. Depth slices of simulated CVES in a 3D environment, with simulated electrode layouts in the $\mathrm{x}$-direction and 5\% noise added. a) model, b) Wenner array, and c) dipole-dipole array. 
MODEL
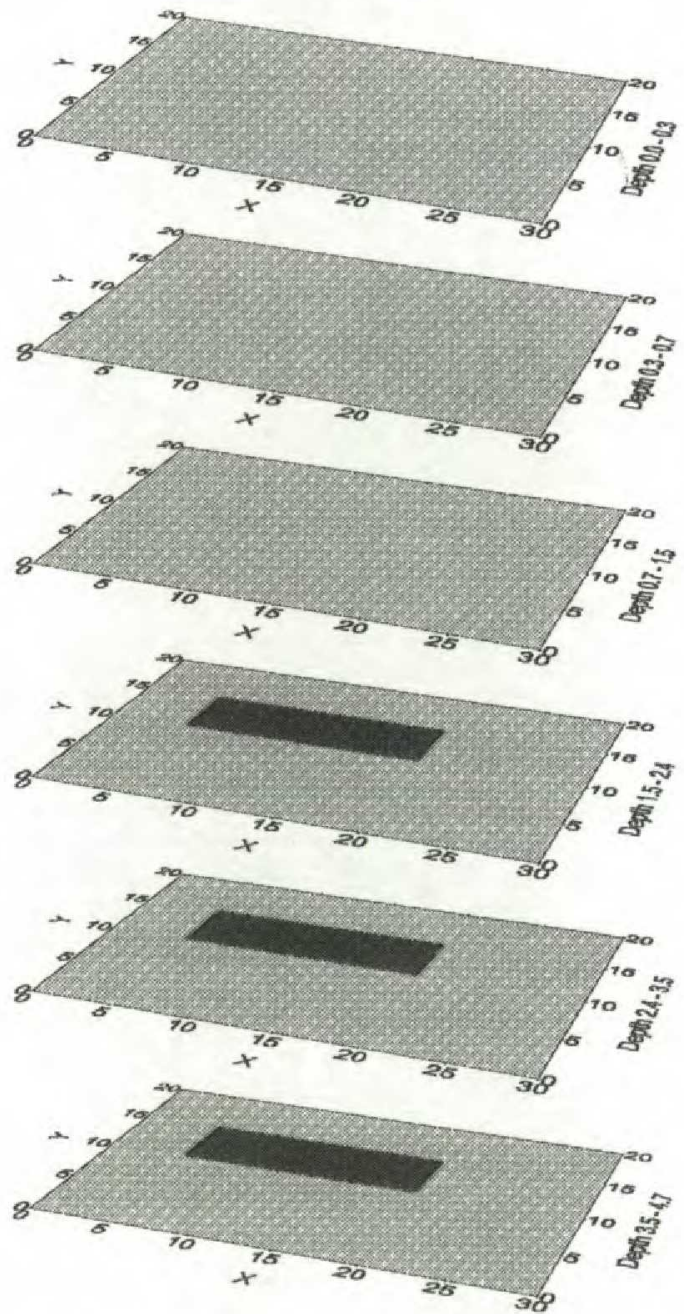

WENNER $3 \mathrm{D}=>2 \mathrm{D}=>3 \mathrm{D}$
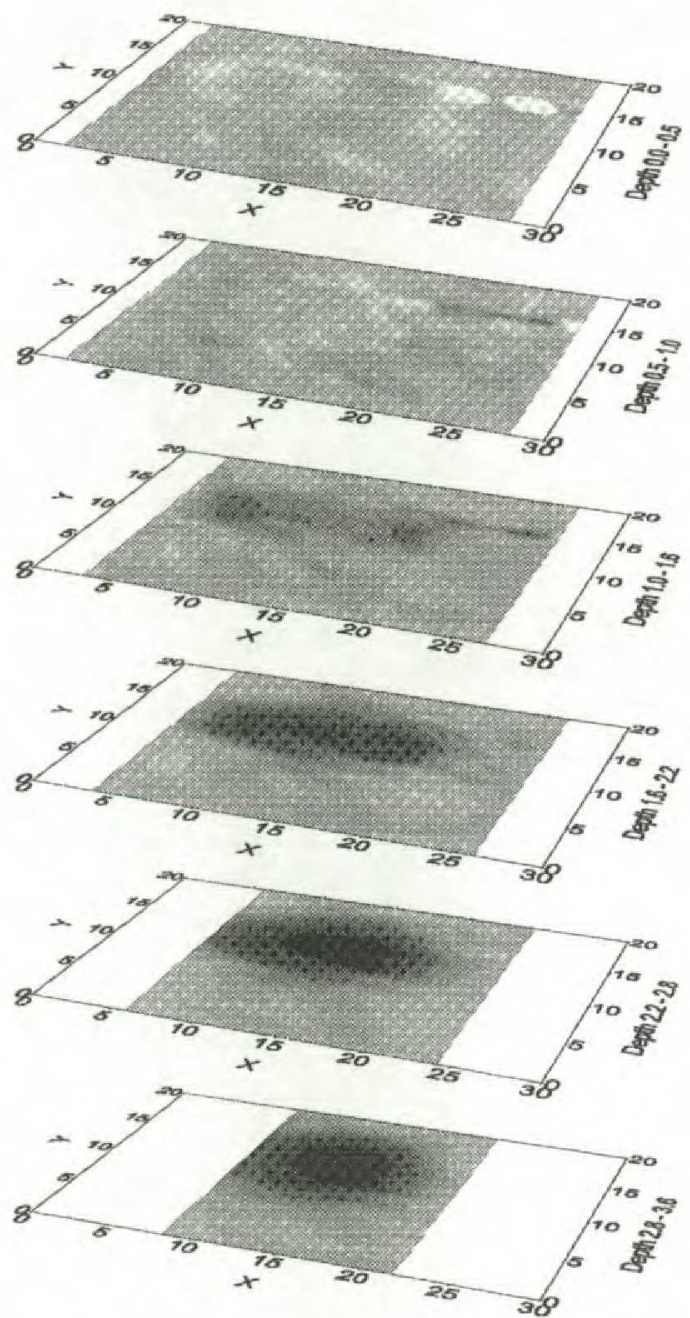

DIPOLE-DIPOLE 3D=>2D=>3D

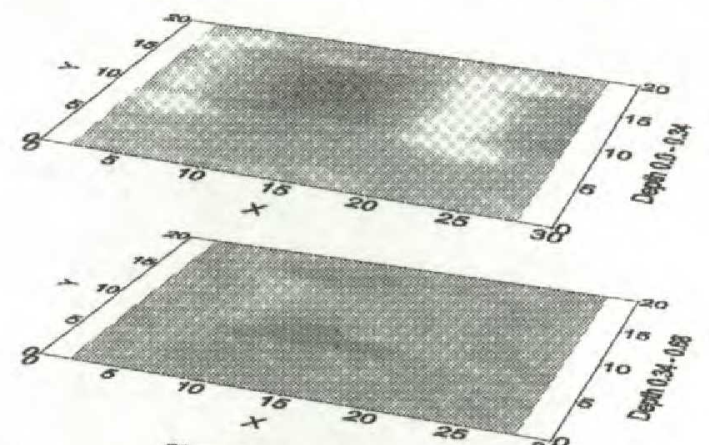

Resistivity!

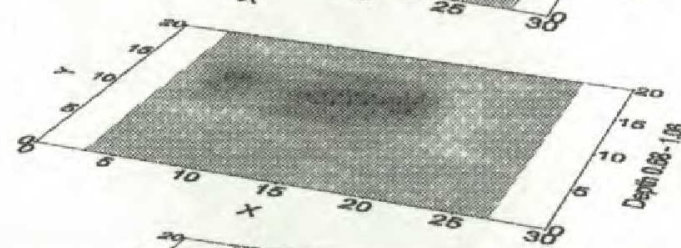

$[\Omega \mathrm{m}]$
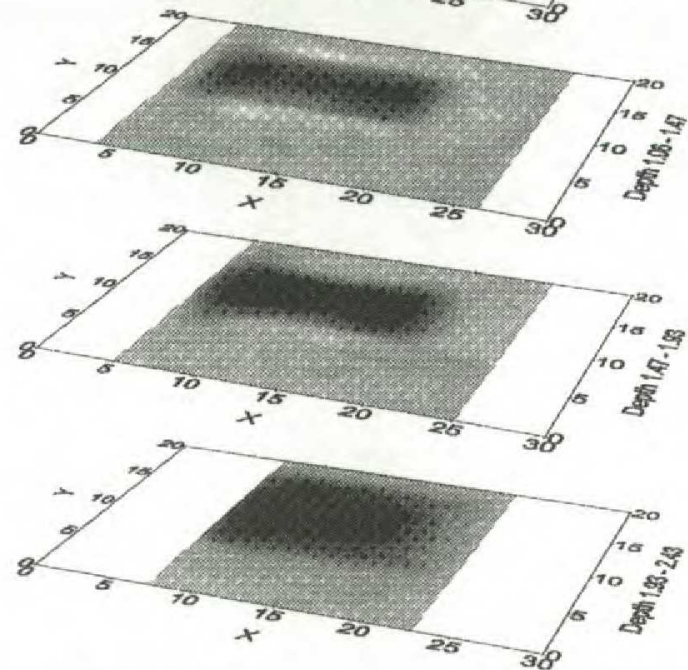

Figure 2. Depth slices of simulated CVES in a 3D environment, with simulated electrode layouts in the x-direction and 5\% noise added. a) model, b) Wenner array, and c) dipole-dipole array. 
resistivity of $100 \Omega \mathrm{m}$. The merged results from the $2 \mathrm{D}$ inversions depicts the structure quite well, the high resistive feature is essentially visible in the two top layers only, but in particular for the dipoledipole array side effects are visible. A significant variation in resistivities in the lower layers is produced as an artefact. The simulated noise creates a variation in all the layers that appear stochastic.

\section{EXAMPLE: LOW RESISTIVE BASIN}

The second example (figure 2) depicts a low resistive basin structure, where a low resistive $(10 \Omega \mathrm{m})$ block appears in a part of the model from the depth level 1.5 down to 7.7. The rest of the model has a resistivity of $100 \Omega \mathrm{m}$. The long direction of the body is in $\mathrm{x}$-direction, i.e. parallel to the profile direction. The inverted result clearly depicts the basin structure. Lateral effects are seen above the low resistive body, especially for the dipole-dipole and pole-dipole arrays. The depth to the top of the low resistive feature is rather well defined for all arrays.

\section{DISCUSSION AND CONCLUSIONS}

The examples above, along with the other models tested, indicate that the quasi-3D technique is a reasonable approach for practical application, since the lateral effects are relatively limited and predictable. The 3D effects in 2D surveying is much less than 2D effects in $1 \mathrm{D}$ surveying, i.e. the step from $1 \mathrm{D}$ to $2 \mathrm{D}$ techniques is more important than the step from $2 \mathrm{D}$ to $3 \mathrm{D}$ techniques.

The magnitude of the effects depend on the electrode array used, where the Wenner array produces the smallest 3D effects and dipole-dipole the largest. Pole-pole and pole-dipole fall in between. Features that are limited in dimension in the profile direction produce rather limited lateral effects, while more elongated features produce more significant effects.

All array types tends to produce shadow effects below anomalous bodies at the surface, especially for low resistive bodies. The effect is largest for the dipole-dipole array but rather small for the Wenner array, which is best at delimiting surficial anomalous bodies towards depth.

The Wenner array does not locate anomalous bodies at depth as distinctly as the other arrays for some tested examples. This may be array dependent, but is probably to some extent related to the small the number of data points calculated by the forward routine compared to the other arrays. The data sets of the other arrays are around three times larger, where the limitations are inherent in the logistics of the array. However, for field data acquired with a single channel instrument the number of data points would probably not be very much larger for any of the other arrays, due to the time consuming measurement procedure. As the number of data points is reduced for the other arrays as well, their resolution power would also decrease accordingly. Furthermore, in real data the sensitivity to noise varies significantly, a very important factor for the resolving power, where the Wenner array is less sensitive to noise than e.g. the dipole-dipole and pole-pole arrays. It would therefore be adequate to apply different noise levels to the different array types in future modelling studies.

\section{ACKNOWLEDGEMENTS}

The work behind this paper has received financial support from Swedish Geological Survey (SGU), Swedish Environmental Protection Agency (AFN/Naturvårdsverket) and Foundation for Technology Transfer in Lund which is gratefully acknowledged.

\section{REFERENCES}

Dey, A. and Morrison, H.F. (1979) Resistivity modelling for arbitrarily shaped three-dimensional structures, Geophysics, vol 44, p 753-780.

Loke, M.H. and Barker, R.D. (1996a) Rapid least-squares inversion of apparent resistivity pseudosections by a quasi-Newton method, Geophysical Prospecting, vol 44, no 1, p 131-152.

Loke, M.H. and Barker, R.D. (1996b) Practical techniques for 3D resistivity surveys and data inversion techniques, Geophysical Prospecting, vol 44, no 3, p 499-524. 